\title{
Improving operating room efficiency-A value proposition
}

\author{
Farhood Farjah, MD, MPH
}

\footnotetext{
From the Division of Cardiothoracic Surgery, and Surgical Outcomes Research Center, University of Washington, Seattle, Wash.

Disclosures: Author has nothing to disclose with regard to commercial support.

Received for publication Jan 14, 2016; accepted for publication Jan 15, 2016; available ahead of print Feb 9, 2016. Address for reprints: Farhood Farjah, MD, MPH, Division of Cardiothoracic Surgery, University of Washington, 1959 NE Pacific St, Box 356310, Seattle, WA 98195 (E-mail: ffarjah@uw.edu).

J Thorac Cardiovasc Surg 2016;151:1396-7

$0022-5223 / \$ 36.00$

Copyright $(2) 2016$ by The American Association for Thoracic Surgery

http://dx.doi.org/10.1016/j.jtcvs.2016.01.023
}

In this issue of the Journal, Azzi and colleagues ${ }^{1}$ report a retrospective study of patients after resection for lung cancer. This study was intended to increase understanding of factors associated with operating time. After adjustment for patient and disease variables, Azzi and colleagues ${ }^{1}$ found that nursing turnover was associated with a significant increase in operating time by nearly an hour. They concluded that active management of the surgical team (eg, staggered nursing shifts and delays in lunch breaks) may be a means of improving operating room (OR) efficiency.

One of the challenges in interpreting a single-institution study is its generalizability. OR processes and structure vary greatly from one hospital to another. Nursing turnover may or may not affect operating times in other settings, where a myriad of other factors may vary from hospital to hospital. Future investigations should measure and adjust for the same variables that Azzi and colleagues ${ }^{1}$ used to facilitate drawing conclusions across studies.

Another challenge in interpreting observational studies is inferring causality. It is plausible that nursing turnover leads to longer operating times, and yet it is equally plausible that longer operating times actually lead to nursing turnover. Retrospectively disentangling the interactions of these variables is difficult, if not impossible. Prospectively defining and measuring these and other variables may help us to understand better the independent effect of nursing turnover on operating time.

As more studies like this one emerge, it will be imperative to perform a systematic review of the literature. The goal of such a review would be to develop a conceptual framework for the relationships between OR efficiency and various patient, disease, provider, and system variables. Qualitative information may supplement quantitative data. Structured interviews or focus groups with key stakeholders-including patients, OR staff, nurses, anesthesiologists, surgeons, administrators, and payers-may shed light on other important variables and their relationships with each other. The purpose of such a conceptual model would be to design multilevel interventions to improve OR efficiency. A multilevel intervention is one that simultaneously changes a patient-level determinant of

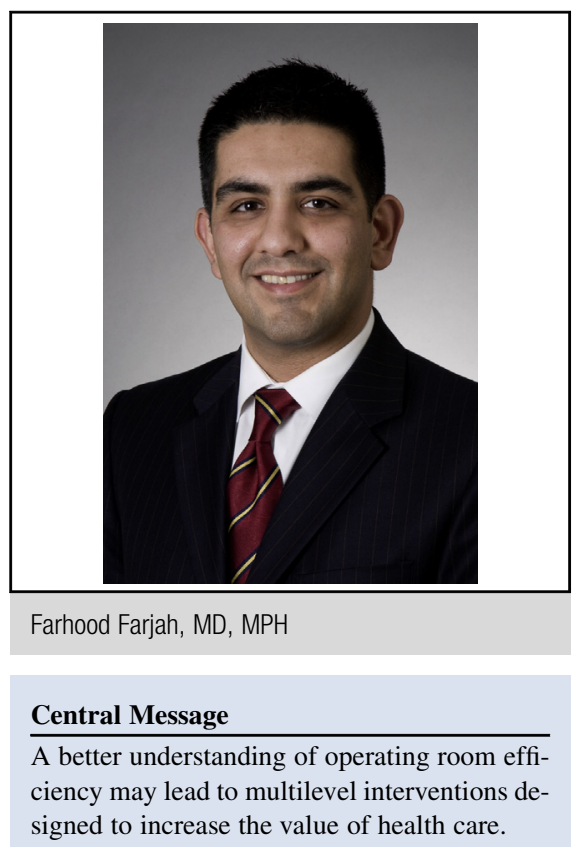

See Article page 1391.

operating times as well as at least 2 other levels of contextual influence, for instance provider behavior or the organizational or practice setting. ${ }^{2}$ Multilevel interventions are more likely to be efficacious and to be accepted by various stakeholders.

The impact of interventions designed to decrease operating time may be assessed in terms of their effect on value. Value can be conceptualized as health outcomes per dollar spent. ${ }^{3}$ Shorter operating times may increase value by improving outcomes and decreasing costs or by decreasing costs without negatively affecting outcomes.

As with any new intervention, it is important first to establish safety. For instance, changing sponge and instrument count protocols during nursing turnover could increase the chances of leaving behind a foreign body. It is also important to demonstrate that an intervention does not lead to other unintended consequences. For example, rescheduling patients to structure a workday for maximum efficiency may lead to patient dissatisfaction. Ways in which shorter operating times may improve outcomes might include by lowering the risk of postoperative adverse events, by increasing the throughput of patients with cancer waiting to undergo curative-intent pulmonary resection, and by improving patient satisfaction by avoiding delays.

To demonstrate that an intervention reduces costs, one must first recognize how costs are measured. Several 
important aspects of a cost analysis include the following: the country in which the operation is performed, because resource costs vary across nations, as do reimbursement structures (single payer, fee for service, etc); the perspective of the analysis (the provider, the payer, or societal); the relative contributions of fixed costs (eg, building, equipment, and salaried workers) and variable costs (eg, supplies and workers paid on an hourly basis); and the population of patients who require care (ie, the volume of services needed within a given geographic region and time period). ${ }^{4}$ Furthermore, because the OR is used by other surgical services to serve various patient populations and other institutional missions (community service), the impact of reducing OR time must be considered in this greater context. Although many of us believe that minutes saved in the OR translate into dollars saved, this belief may or may not be true under various circumstances.
The challenges associated with understanding and optimizing OR efficiency should not be perceived as a barrier to further progress. Instead, these challenges should be viewed as research and career development opportunities for the intrepid. Azzi and colleagues ${ }^{1}$ are to be congratulated for their efforts to increase our knowledge of factors that affect OR time, and for advancing research that may increase the value of health care.

\section{References}

1. Azzi AJ, Shah K, Seely A, Villeneuve JP, Sundaresan SR, Shamji FM, et al Surgical team turnover and operative time: an evaluation of operating room efficiency during pulmonary resection. J Thorac Cardiovasc Surg. 2016;151: 1391-5.

2. Taplin SH, Anhang Price R, Edwards HM, Foster MK, Breslau ES, Chollette V, et al. Introduction: understanding and influencing multilevel factors across the cancer care continuum. J Natl Cancer Inst Monogr. 2012;44:2-10.

3. Porter ME. What is value in health care? N Engl J Med. 2010;363:2477-81.

4. Macario A. What does one minute of operating room time cost? J Clin Anesth. 2010;22:233-6. 